Tema: Aciaria Elétrica

\title{
OTIMIZAÇÃO DA ENERGIA QUÍMICA EM CARGAS COM E SEM GUSA LÍQUIDA NO FEA DA SINOBRAS UTILIZANDO A METODOLOGIA DOE*
}

\author{
Neiclésio Nunes Ibiapina ${ }^{1}$ \\ Silvio Cesar de Oliveira ${ }^{2}$ \\ Rubens José Giusti de Arruda ${ }^{3}$
}

\begin{abstract}
Resumo
No forno elétrico a arco (FEA) é realizado a fusão da carga metálica composta de sucata, gusa sólido ou líquido, fundentes e o aquecimento do banho líquido utilizando energia elétrica e química. O balanço correto dessas energias e a escolha adequada das matérias primas são fatores que determinam a otimização da eficiência energética e demais ganhos advindos. Para melhoria desses fatores foi utilizada a metodologia Delineamento de Experimentos (DOE) que permite estudar as variáveis do processo e a complexidade de suas interações, buscando solucionar problemas e melhorar a desempenho através de análises estatísticas. Este trabalho mostra os experimentos realizados no FEA da Sinobras objetivando otimizar a energia química do FEA para diferentes condições de carga. O resultado alcançado foi a redução no consumo de energia elétrica, no consumo de oxigênio, no power on e aumento da produção.
\end{abstract}

Palavras-chave: Forno elétrico a arco; DOE; Injeção de oxigênio; Eletricidade.

\section{OPTIMIZATION OF CHEMICAL ENERGY IN CARGO NET AND WITHOUT IRON IN THE SINOBRAS FEA USING A METHODOLOGY DOE}

\begin{abstract}
In the electric Arc furnace (EAF) is carried melting the metal charge composed of scrap, pig iron or hot metal, fluxes and the liquid steel heating using electrical and chemical energy. The correct balance of these energies and proper choice of raw materials are factors that determine the optimization of energy efficiency and other gains. To improve these factors the Design of Experiments (DOE) was used to study the process variables and the complexity of their interactions, seeking to solve problems and improve performance through statistical analysis. This work presents the experiments performed in the Sinobras EAF aiming at optimizing the chemical energy for different charge conditions. The result achieved was a reduction in electricity consumption, oxygen consumption, the power on time and increased production.
\end{abstract}

Keywords: Electric furnace; DOE; Oxygen injection; Electricity.

Engenheiro de Materiais, Engenheiro de Processos, Sinobras, Marabá, PA, Brasil.

Engenheiro Mecânico, Gerente da aciaria, Sinobras, Marabá, PA, Brasil.

Químico, Assessor Técnico, Sinobras, Marabá, PA, Brasil.

* Contribuição técnica ao $45^{\circ}$ Seminário de Aciaria - Internacional, 25 a 28 de maio de 2014, Porto Alegre, RS, Brasil. 


\section{INTRODUÇÃO}

O aumento da produção de aço utilizando forno elétrico a arco (FEA) ao redor do mundo tem se direcionado para baixos investimentos e alta flexibilidade operacional no que se diz respeito à carga metálica utilizada, busca do aumento da produtividade e redução do consumo de energia elétrica entre outros indicadores importantes. Com o aumento da competitividade e a busca por melhor eficiência nos processos, vários desenvolvimentos vem sendo realizados.

No FEA o principal objetivo é fundir a carga metálica e elevar sua temperatura até aproximadamente 1.600 e $1.650^{\circ} \mathrm{C}$ para realizar o vazamento da corrida, este processo acontece utilizando energia química e elétrica.

$\mathrm{Na}$ Figura 1 é possível observar o balanço energético dos novos fornos que geralmente utilizam aproximadamente $65 \%$ de energia elétrica, $5 \%$ da energia dos queimadores e $30 \%$ é a energia proveniente das reações químicas.

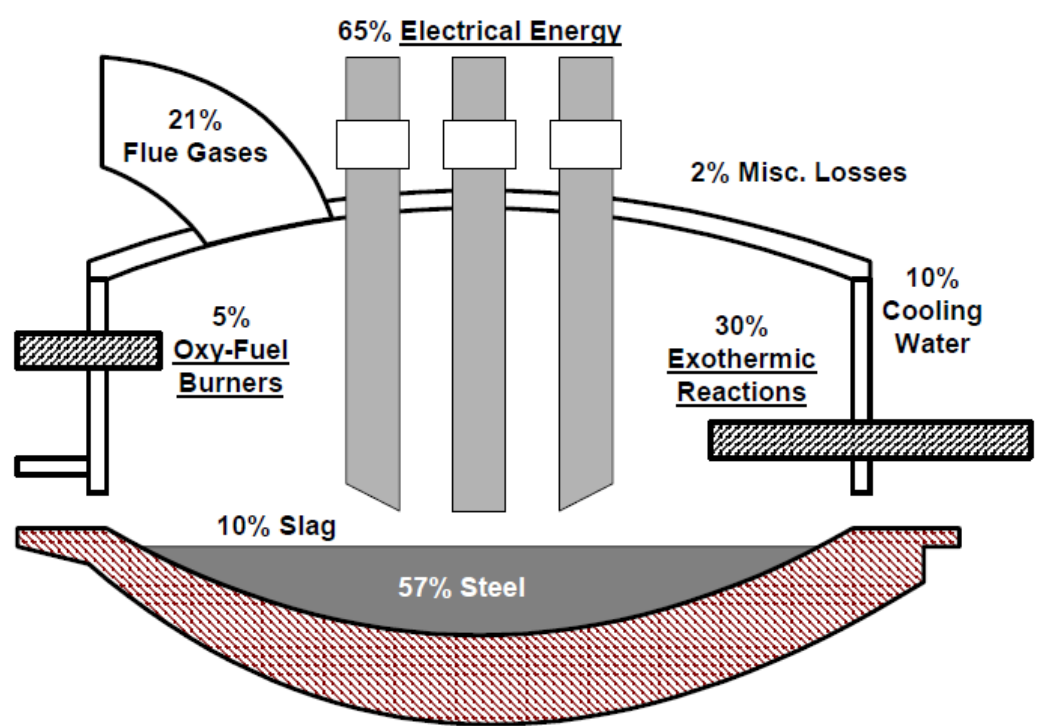

Figura 1. Balanço energético típico do FEA [1].

Segundo Pretorius [2] com a equação abaixo é possível calcular o calor necessário (entalpia) para aquecer o ferro da temperatura ambiente $\left(25^{\circ} \mathrm{C}\right)$ até a $\mathrm{T}$ onde $\mathrm{o}$ mesmo já está fundido.

$$
\text { Energia }(\mathrm{kWh} / \mathrm{Kg} \mathrm{Fe})=\left(2.27 \times 10^{-4} * \mathrm{~T}\right)+0,0142
$$

Com essa equação é possível calcular, por exemplo, a energia requerida para fundir 1 tonelada de $\mathrm{Fe}$ puro de $25^{\circ} \mathrm{C}$ até $1.650^{\circ} \mathrm{C}$ que é $389 \mathrm{kWh}$.

Nos FEA a energia química é oriunda dos elementos queimadores ou das reações exotérmicas. O oxigênio reage com o silício, carbono, manganês, hidrocarbonetos e o ferro, esses elementos originam de queimadores, injeção de carbono e de constituintes da carga de sucata e gusa líquida.

A tabela abaixo mostra as mais importantes reações exotérmicas que ocorrem no FEA a $1.650^{\circ} \mathrm{C}$.

* Contribuição técnica ao 450 Seminário de Aciaria - Internacional, 25 a 28 de maio de 2014, Porto Alegre, RS, Brasil. 


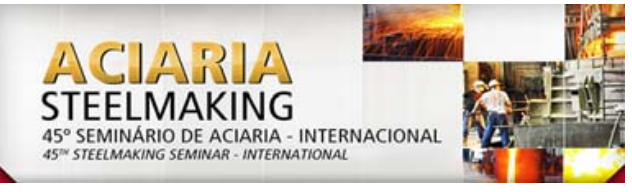

Tabela 1. Reações exotérmicas que ocorrem no FEA [2]

\begin{tabular}{|c|c|}
\hline Reação & $\begin{array}{c}\Delta \mathrm{H} \\
(\mathrm{kWh} / \mathrm{kg})\end{array}$ \\
\hline $\mathrm{Fe}+1 / 2 \mathrm{O}_{2}(\mathrm{~g})=\mathrm{FeO}$ & -1.275 \\
\hline $\mathrm{Mn}+1 / 2 \mathrm{O}_{2}=\mathrm{MnO}$ & -2.044 \\
\hline $4 \mathrm{Al}+3 \mathrm{O}_{2}(\mathrm{~g})=2 \mathrm{Al}_{2} \mathrm{O}_{3}$ & -8.650 \\
\hline $\mathrm{C}+1 / 2 \mathrm{O}_{2}(\mathrm{~g})=\mathrm{CO}(\mathrm{g})$ & -2.739 \\
\hline $\mathrm{CO}(\mathrm{g})+1 / 2 \mathrm{O}_{2}(\mathrm{~g})=\mathrm{CO}(\mathrm{g})_{2}$ & -2.763 \\
\hline $\mathrm{C}+\mathrm{O}_{2}(\mathrm{~g})=\mathrm{CO}(\mathrm{g})_{2}$ & -9.184 \\
\hline $\mathrm{H}_{2}(\mathrm{~g})+1 / 2 \mathrm{O}_{2}(\mathrm{~g})=\mathrm{H}_{2} \mathrm{O}(\mathrm{g})$ & -34.614 \\
\hline $\mathrm{Si}+\mathrm{O}_{2}(\mathrm{~g})=\mathrm{SiO}_{2}$ & -9.348 \\
\hline $\mathrm{CH}_{4}(\mathrm{~g})+2 \mathrm{O}_{2}(\mathrm{~g})=\mathrm{CO}_{2}(\mathrm{~g})+\mathrm{H}_{2} \mathrm{O}(\mathrm{g})$ & \\
\hline
\end{tabular}

Segundo Bihel [3], durante o processo também é necessário controlar a basicidade da escória formada adicionando cal calcítica e dolomita para manter a relação $\left(\mathrm{CaO} /\left[\mathrm{SiO}_{2}+\mathrm{Al}_{2} \mathrm{O}_{3}\right]\right)$ entre 2,0 e 2,4 e formar uma escória "espumante" que aumenta a eficiência da energia elétrica adicionada durante o refino.

\subsection{Energia dos Queimadores}

Segundo Chevrand [4] os queimadores agora estão presentes em quase todos os fornos ao redor do mundo e estes são colocados nos "pontos frios" que estão ilustrados em marrom na Figura 2. São pontos onde o arco elétrico proveniente dos eletrodos possui baixa eficiência e com as instalações dos queimadores nestes locais o resultado é uma fusão uniforme da carga, evitando assim queda de sucata e consequentemente à quebra de eletrodo, assim como e a redução do tempo de forno ligado necessário, reduzindo também o consumo de eletrodos e eletricidade.

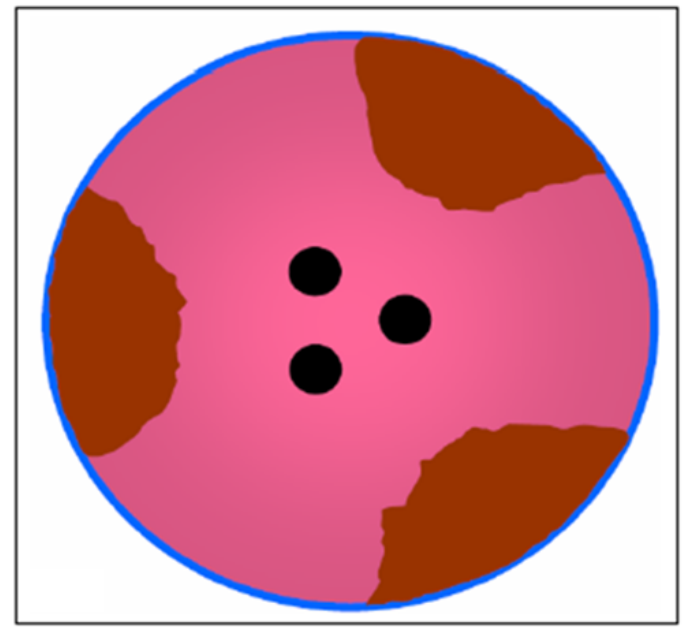

Figura 2. llustração dos pontos frios do FEA [5].

\subsection{Injeção de Oxigênio}

No FEA acontece o refino primário do aço que consiste na retirada de impurezas por meio da sua oxidação. Essa oxidação é realizada através da injeção de oxigênio no forno por meio de injetores posicionados estrategicamente. Segundo Hopperdiezel [6] como os óxidos que são formados possuem menor densidade que o banho, eles sobem para a escória e são retirados através da porta de escória, porém juntamente

* Contribuição técnica ao 45 Seminário de Aciaria - Internacional, 25 a 28 de maio de 2014, Porto Alegre, RS, Brasil. 


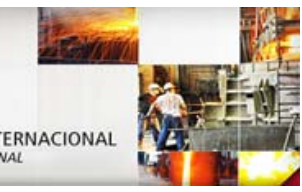

com essa oxidação de elementos indesejáveis, ocorre a oxidação do $\mathrm{Fe}$ o qual também vai para escória e é retirado na forma de $\mathrm{FeO}$, porém, além do oxigênio, em geral, um material carbonáceo é injetado na interface metal/escória, nesse processo o carbono reage com o $\mathrm{FeO}$ produzindo $\mathrm{CO}$ conforme a reação abaixo, que representa a formação da escória espumante que protege o arco elétrico e aumenta a eficiência térmica e energética do processo.

$$
\mathrm{FeO}+\mathrm{C}_{(\mathrm{s})} \rightarrow \mathrm{CO}_{(\mathrm{g})}+\mathrm{Fe}
$$

O processo de injeção de oxigênio também fornece energia ao processo através das reações exotérmicas dos elementos presentes na carga como carbono, silício, manganês e ferro que ao reagir com oxigênio geram energia química adicional. $A$ Figura 3 mostra a efeito do oxigênio na redução do consumo de energia elétrica.

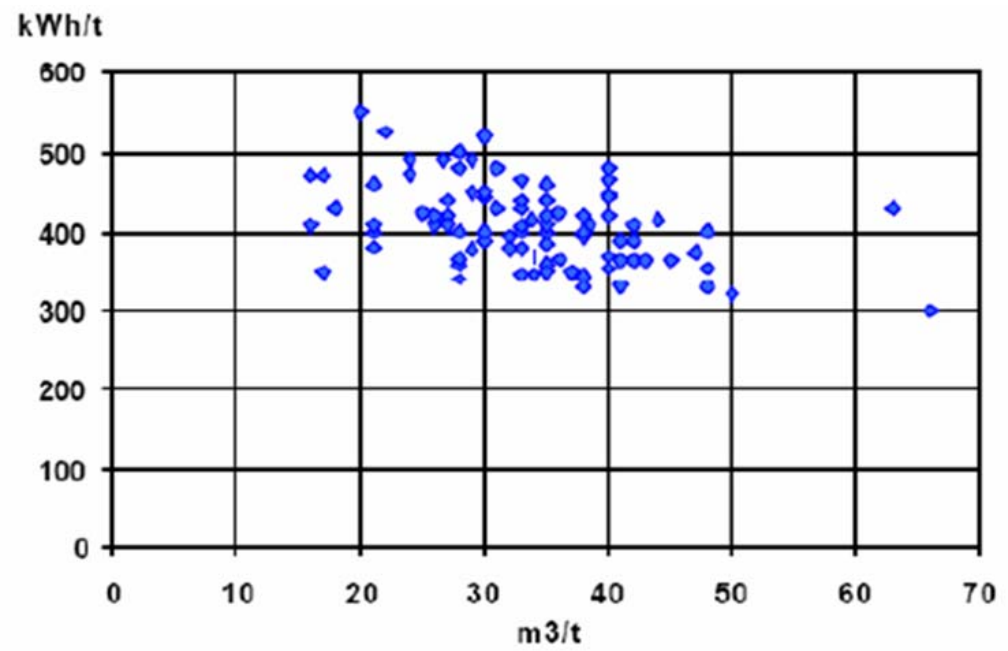

Figura 3. Relação entre a quantidade de oxigênio versus o consumo de energia elétrica [7].

Segundo Gaskell [8] durante o refino é importante também determinar o mínimo de oxigênio que deve ser injetado para realizar a combustão completa do carbono e $\mathrm{CO}$, porém, essa quantidade deve ser controlada de forma precisa para evitar também a excessiva oxidação do $\mathrm{Fe}$ para $\mathrm{FeO}$ e consequentemente à redução do rendimento metálico, a Figura 4 mostra a relação existente entre o teor de carbono no banho, teor de $\mathrm{FeO}$ na escória e a concentração de oxigênio no banho.

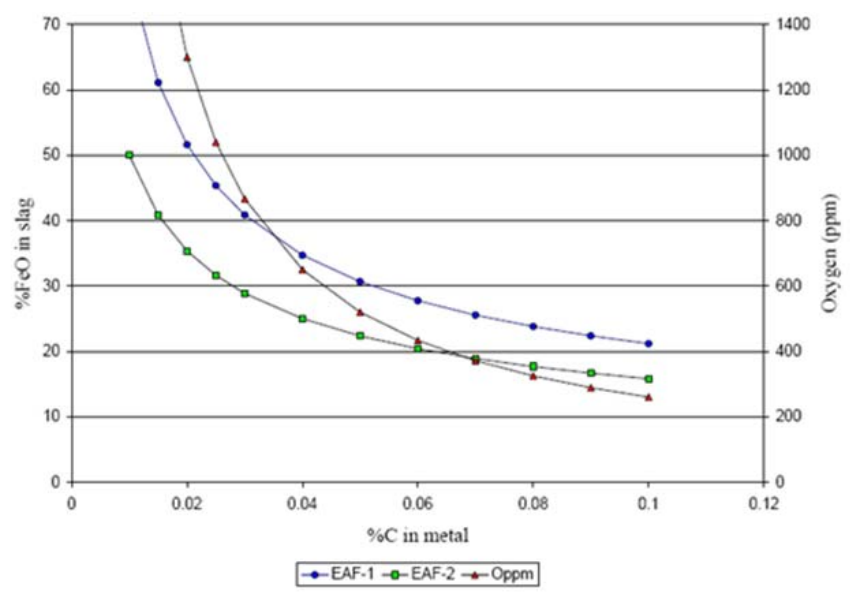

Figura 4. Relação entre o \%FeO na escória, \%C no banho e (PPM) de oxigênio [2].

* Contribuição técnica ao 450 Seminário de Aciaria - Internacional, 25 a 28 de maio de 2014, Porto Alegre, RS, Brasil. 
Na Sinobras o oxigênio pode ser adicionado no FEA por meio de um robô manipulador que possui tubos consumíveis que, além de cortar sucata, injetam oxigênio no banho através da porta de escória ou por injetores supersônicos que possuem, através de tecnologia, uma lança virtual para formar um jato coerente penetre no banho líquido.

\subsection{Energia Elétrica no FEA}

Na teoria, são necessários no mínimo $300 \mathrm{kWh} / \mathrm{t}$ para fundir e aquecer o banho até a temperatura de vazamento, porém esses consumos mudam com o tipo de carga, prática operacional e perdas do sistema, geralmente a energia elétrica consumida nos fornos atuais varia entre 340 a $500 \mathrm{kWh} / \mathrm{t}$. Esta variação acontece devido aos custos das matérias primas e insumos, dos investimentos em novas tecnologias que aumentam a eficiência dos equipamentos, das práticas utilizadas em cada processo, etc. Em aciarias onde o custo de energia elétrica é elevado, por exemplo, é vantajoso utilizar a energia química como alternativa para redução do custo da produção do aço.

\subsection{Design of Experiment}

Design of Experiment (DOE) é uma técnica utilizada para se planejar experimentos, ou seja, para definir quais dados, quantidade e condições devem ser coletados durante um determinado experimento, buscando, basicamente, satisfazer dois grandes objetivos que são a realização do experimento com menor custo e maior precisão estatística possível na resposta.

Em geral, os experimentos são utilizados para estudar o desempenho de processos que podem ser considerados com uma combinação de elementos que transformam entrada em uma saída que tem uma ou mais respostas observáveis.

Existem diversos softwares como, por exemplo, o Minitab® que auxilia na criação e análise de experimentos do tipo DOE, com ele é possível, após a seleção da combinação dos fatores, executar as combinações que permite analisar os resultados dos dados de entrada-saída do experimento, para se gerar conclusões sobre a importância dos fatores.

\section{MATERIAL E MÉTODOS}

O FEA da Sinobras tem capacidade de $40 \mathrm{t}$ de aço líquido por corrida, possui 3 queimadores/injetores e duas lanças consumíveis utilizadas através de robô manipulador. Para realização do experimento foram utilizados 4 programas de injeção de oxigênio no FEA estequiometricamente calculados para cargas com gusa líquida e sem gusa líquida.

O objetivo foi analisar, através da ferramenta DOE, a influência da vazão de oxigênio dos injetores e o tempo de injeção nas variáveis de saída do processo como teor de carbono no aço, teor de FeO na escória, consumo de energia elétrica e tempo de forno ligado (power on).

\subsection{Características do Forno}

A Tabela 2 possui os dados do forno elétrico a arco onde o experimento foi realizado.

\footnotetext{
* Contribuição técnica ao 45 Seminário de Aciaria - Internacional, 25 a 28 de maio de 2014, Porto Alegre, RS, Brasil.
} 
Tabela 2. Dados do Forno Elétrico da Sinobras

\begin{tabular}{|c|c|}
\hline Fabricante & $\mathrm{BSE}$ \\
\hline Volume do forno & $57 \mathrm{~m}^{3}$ \\
\hline Capacidade do forno & $44 \mathrm{t}$ \\
\hline Tipo de forno & EBT \\
\hline Diâmetro dos eletrodos & $18 "$ \\
\hline
\end{tabular}

\subsection{Composição da Carga}

Nas Tabelas 3, 4 e 5, esta a composição química média da carga adicionada no FEA durante o experimento. As quantidades adicionadas de sucata, gusa líquida e fundentes foram fixas para cada etapa do experimento.

Tabela 3. Média da composição química da gusa líquida

\begin{tabular}{|c|c|c|c|c|}
\hline \%C & \%Si & \%Mn & \%S & \%P \\
\hline 3,00 & 0,30 & 0,50 & 0,20 & 0,30 \\
\hline
\end{tabular}

Tabela 4. Média da composição química da sucata

\begin{tabular}{|c|c|c|c|c|}
\hline \%C & \%Si & \%Mn & \%S & \%P \\
\hline 0,10 & 0,15 & 0,20 & 0,15 & 0,20 \\
\hline
\end{tabular}

Tabela 5. Média da composição dos fundentes

\begin{tabular}{|c|c|c|}
\hline Tipo & \%CaO & \%MgO \\
\hline Cal Calcítica & 94,00 & - \\
\hline Dolomita & 50,00 & 32,00 \\
\hline
\end{tabular}

\subsection{Programas de Injeção de Oxigênio}

Abaixo as Tabelas 6 a 9 mostram os programas de injeção utilizados no experimento.

Tabela 6. Programa de injeção 1, sem gusa líquida

\begin{tabular}{|c|c|c|c|}
\hline \multicolumn{4}{|c|}{ Programa 1 - Sem Gusa Líquida } \\
\hline Injetor & Vazão $\left(\mathrm{Nm}^{3} / \mathrm{h}\right)$ & Pressão (bar) & Tempo (min.) \\
\hline VLB 1 & 1300 & 14 & 15 \\
\hline VLB 2 & 1300 & 14 & 15 \\
\hline VLB 3 & 1300 & 14 & 15 \\
\hline Lança & 1600 & 14 & 23 \\
\hline
\end{tabular}

Tabela 7. Programa de injeção 2, sem gusa líquida

\begin{tabular}{|c|c|c|c|}
\hline \multicolumn{4}{|c|}{ Programa 2 - Sem Gusa Líquida } \\
\hline Injetor & Vazão $\left(\mathrm{Nm}^{3} / \mathrm{h}\right)$ & Pressão (bar) & Tempo (min.) \\
\hline VLB 1 & 1600 & 14 & 12,2 \\
\hline VLB 2 & 1600 & 14 & 12,2 \\
\hline VLB 3 & 1600 & 14 & 12,2 \\
\hline Lança & 1600 & 14 & 23 \\
\hline
\end{tabular}

Tabela 8. Programa de injeção 3, com gusa líquida

\begin{tabular}{|c|c|c|c|}
\hline \multicolumn{4}{|c|}{ Programa 3 - Com Gusa Líquida } \\
\hline Injetor & Vazão $\left(\mathrm{Nm}^{3} / \mathrm{h}\right)$ & Pressão (bar) & Tempo (min.) \\
\hline VLB 1 & 1300 & 14 & 16 \\
\hline VLB 2 & 1300 & 14 & 16 \\
\hline VLB 3 & 1300 & 14 & 16 \\
\hline Lança & 1600 & 14 & 23 \\
\hline
\end{tabular}

* Contribuição técnica ao 45 Seminário de Aciaria - Internacional, 25 a 28 de maio de 2014, Porto Alegre, RS, Brasil. 
Tabela 9. Programa de injeção 4, com gusa líquida

\begin{tabular}{|c|c|c|c|}
\hline \multicolumn{4}{|c|}{ Programa 4 - Com Gusa Líquida } \\
\hline Injetor & Vazão $\left(\mathrm{Nm}^{3} / \mathrm{h}\right)$ & Pressão (bar) & Tempo (min.) \\
\hline VLB 1 & 1600 & 14 & 14 \\
\hline VLB 2 & 1600 & 14 & 14 \\
\hline VLB 3 & 1600 & 14 & 14 \\
\hline Lança & 1600 & 14 & 23 \\
\hline
\end{tabular}

\subsection{Experimento}

O experimento foi projetado utilizando o software Minitab $15 \mathrm{com}$ a ferramenta Stat $\rightarrow D O E$-> Factorial. E foi escolhido, tanto para gusa líquida, quanto carga sem gusa o tipo de projeto 2-level factorial com 2 fatores conforme a Figura 5.

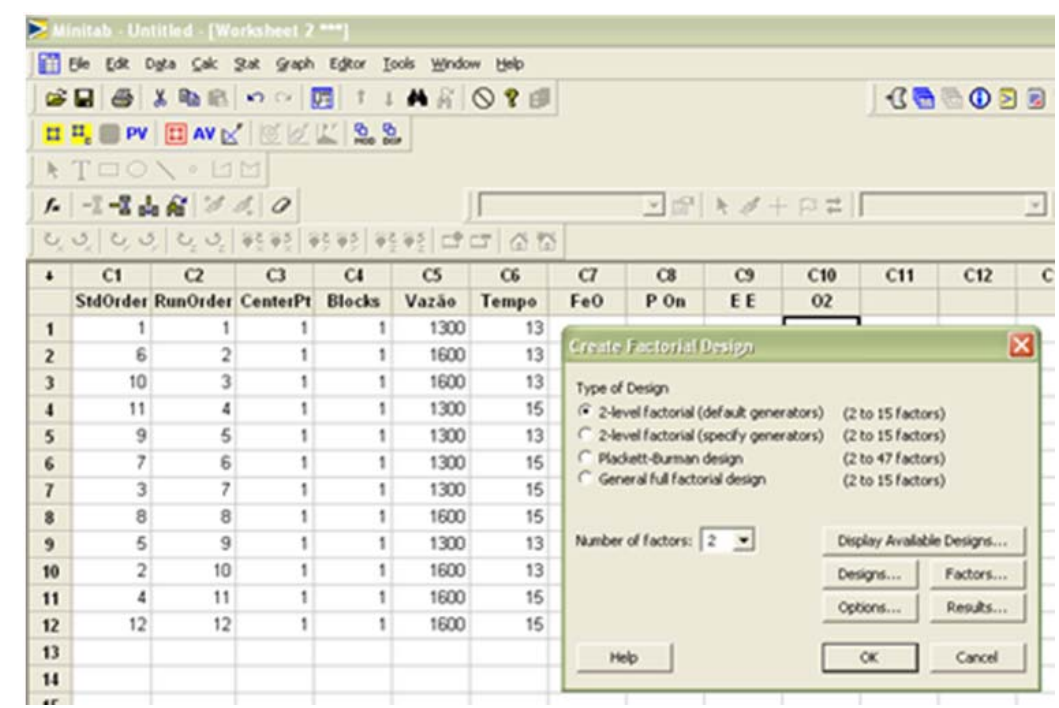

Figura 5. Criação do experimento.

O software gerou o experimento com três réplicas e a ordem que o experimento deve ser realizado.

Para o experimento foi utilizado o mesmo tipo e quantidade de sucata e gusa líquida. Os resultados foram anotados e inseridos no software para posterior análise.

\section{RESULTADOS E DISCUSSÕES}

\subsection{Resultados da Interação Entre os Fatores}

Após a realização dos experimentos os dados foram copilados no software Minitab onde foi analisada a interação entre os fatores através da função Stat - >DOE - > Factorial - > Analyze Factorial Design.

As Figuras 6 a 9 mostram os resultados através dos gráficos de Pareto para a interação entre as variáveis de saída e a vazão de oxigênio e o tempo em corridas sem utilizar gusa líquida.

\footnotetext{
* Contribuição técnica ao $45^{\circ}$ Seminário de Aciaria - Internacional, 25 a 28 de maio de 2014 , Porto Alegre, RS, Brasil.
} 

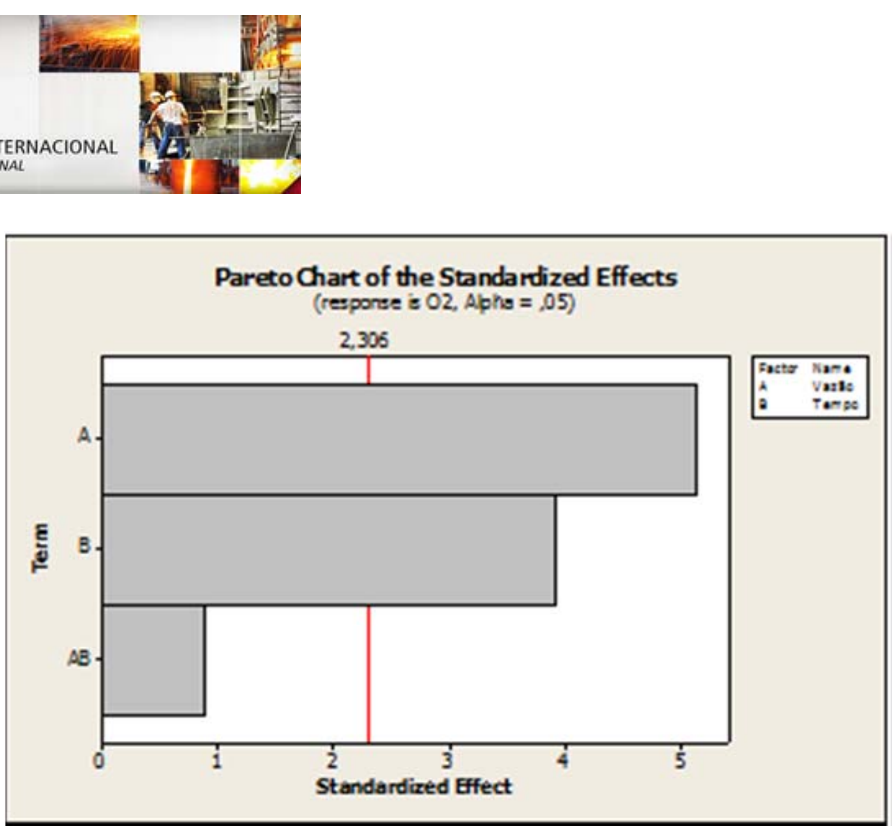

Figura 6. Pareto do efeito da interação entre o consumo de oxigênio, a vazão de oxigênio e o tempo de injeção.

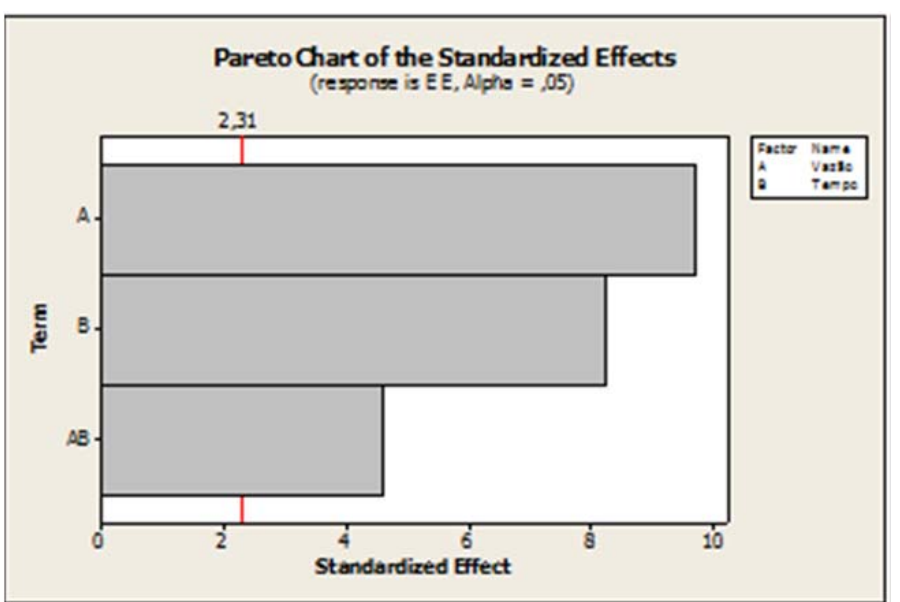

Figura 7. Pareto do efeito da interação entre a energia elétrica, a vazão de oxigênio e o tempo de injeção.

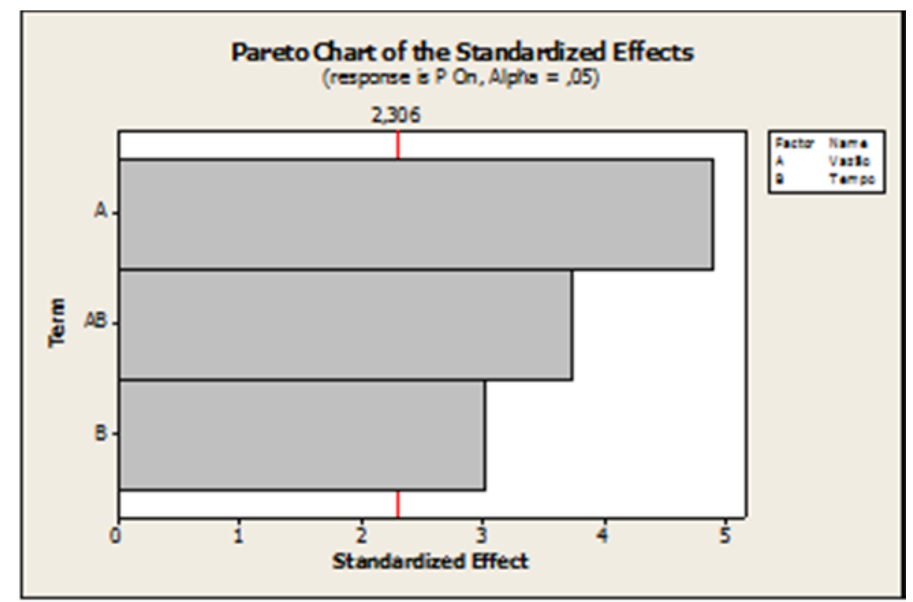

Figura 8. Pareto do efeito da interação entre o power on, a vazão de oxigênio e o tempo de injeção.

* Contribuição técnica ao $45^{\circ}$ Seminário de Aciaria - Internacional, 25 a 28 de maio de 2014, Porto Alegre, RS, Brasil. 


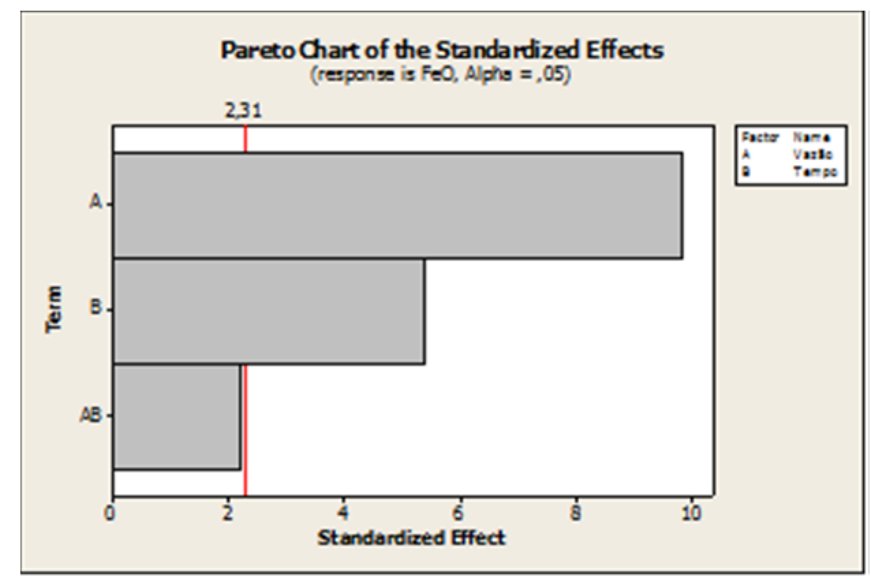

Figura 9. Pareto do efeito da interação entre o $\mathrm{FeO}$ da escória, a vazão de oxigênio e o tempo de injeção.

Ao nível de significância de 95\%, existe interação significativa entre a vazão de oxigênio e o tempo de injeção para o consumo total de oxigênio e teor de FeO na escória. Para o consumo de energia elétrica e power on, existe interação significativa entre a vazão de oxigênio e o tempo de injeção além da combinação entre esses dois fatores. As Figuras 10 a 13 mostram os resultados através dos gráficos de Pareto para a interação entre as variáveis de saída e a vazão de oxigênio e o tempo em corridas utilizando gusa líquida.

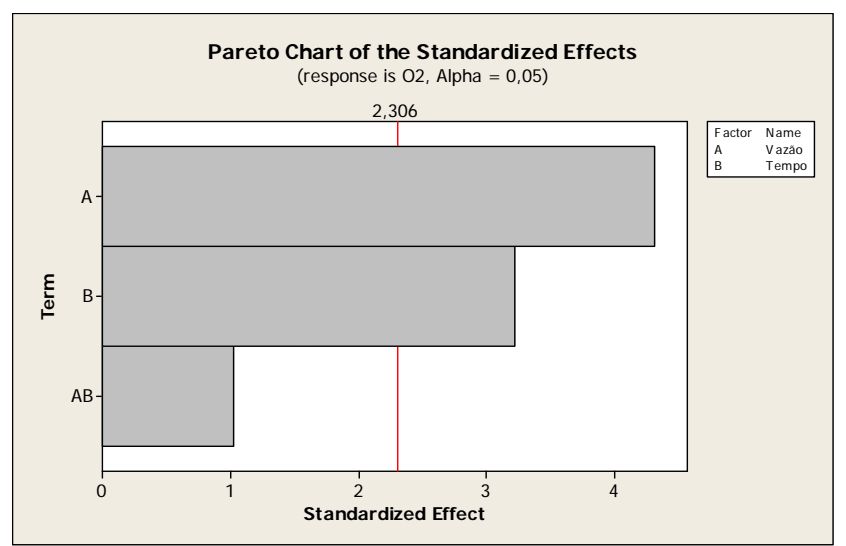

Figura 10. Pareto do efeito da interação entre o consumo de oxigênio, a vazão de oxigênio e o tempo de injeção.

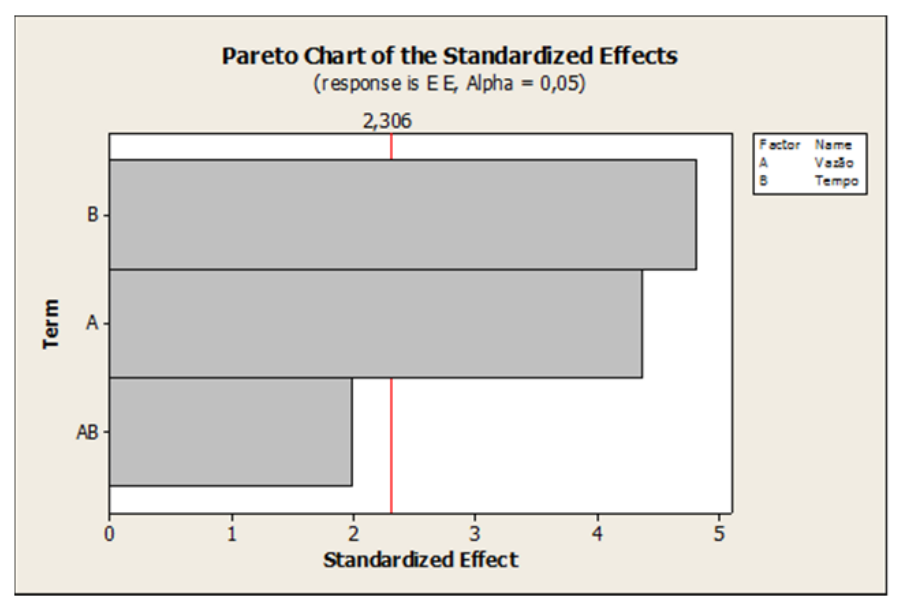

Figura 11. Pareto do efeito da interação entre a energia elétrica, a vazão de oxigênio e o tempo de injeção.

* Contribuição técnica ao 45 Seminário de Aciaria - Internacional, 25 a 28 de maio de 2014, Porto Alegre, RS, Brasil. 

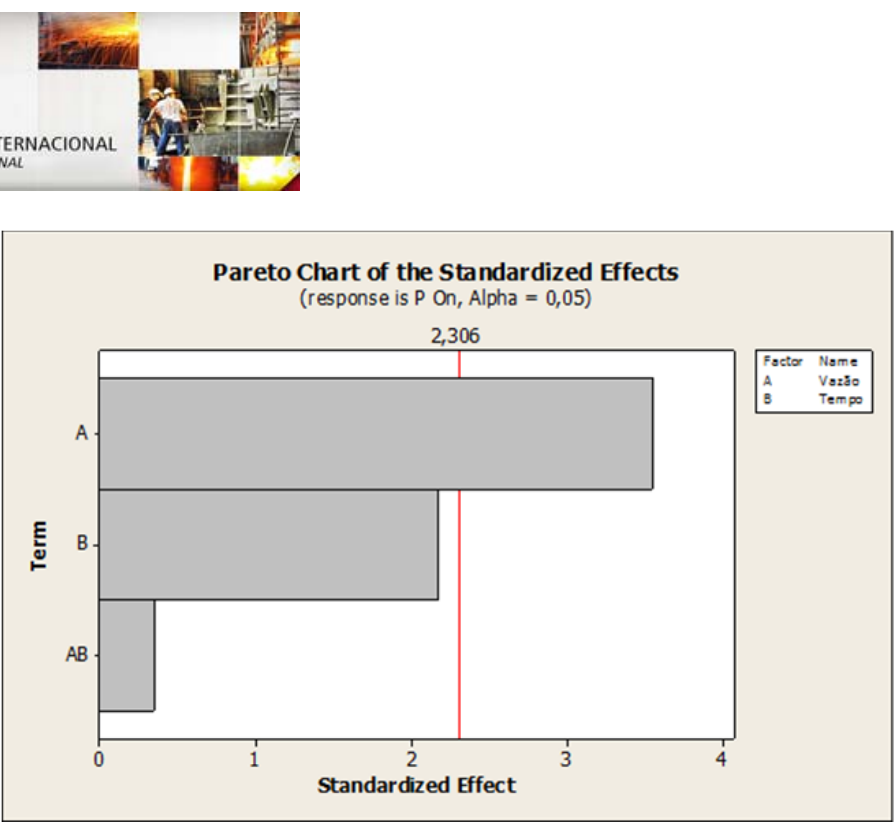

Figura 12. Pareto do efeito da interação entre o power on, a vazão de oxigênio e o tempo de injeção.

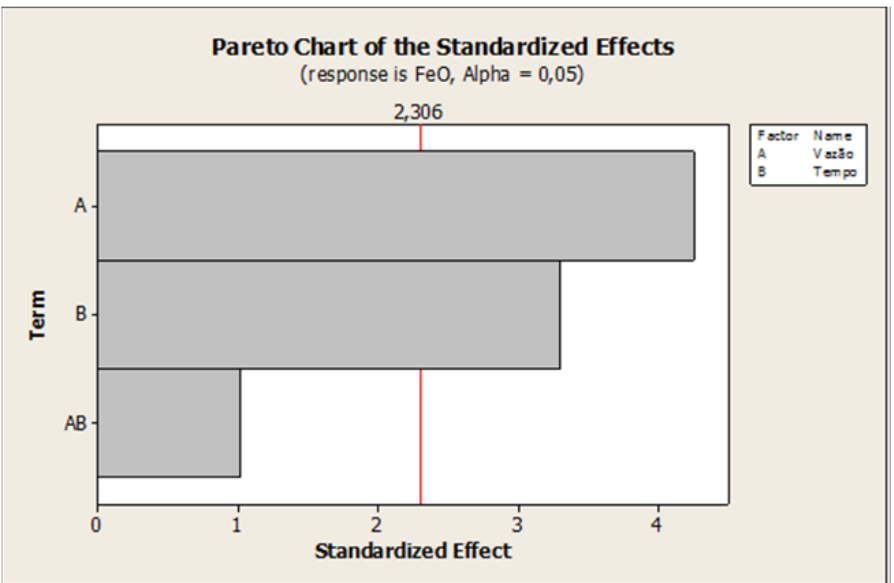

Figura 13. Pareto do efeito da interação entre o $\mathrm{FeO}$ da escória, a vazão de oxigênio e o tempo de injeção.

\subsection{Resultados da Otimização}

Após a análise de interação foi feito através do Minitab pela função Stat -> DOE -> Factorial $\rightarrow$ Response Optimizer a simulação, utilizando parâmetros preestabelecidos da condição de $\mathrm{FeO}$, power on e consumo de energia elétrica desejados. Nas Figuras 14 e 15 são apresentados os valores otimizados de vazão de oxigênio e tempo de injeção para corridas sem gusa líquida e com gusa líquida respectivamente.

\begin{tabular}{||c||cc|}
\hline Optimal & Vazão & Tempo \\
$D$ & 1600,0 & 15,0 \\
D Cur & {$[1450,0]$} & {$[14,0]$} \\
0,00000 Low & 1300,0 & 13,0 \\
\hline
\end{tabular}

Figura 14. Valor de vazão de oxigênio e tempo de injeção otimizado para corridas sem gusa líquida.

\begin{tabular}{|c|c|c|}
\hline $\begin{array}{cc}\text { Optimal } & \text { High } \\
\text { D } & \text { Cur } \\
0,00000 & \text { Low }\end{array}$ & $\begin{array}{c}\text { Vazão } \\
1600,0 \\
{[1600,0]} \\
1300,0\end{array}$ & $\begin{array}{c}\text { Tempo } \\
16,0 \\
{[16,0]} \\
14,0\end{array}$ \\
\hline
\end{tabular}

Figura 15. Valor de vazão de oxigênio e tempo de injeção otimizado para corridas com gusa líquida.

Os resultados obtidos na otimização foram testados, a seguir os resultados.

* Contribuição técnica ao $45^{\circ}$ Seminário de Aciaria - Internacional, 25 a 28 de maio de 2014, Porto Alegre, RS, Brasil. 


\subsection{Resultados dos Testes}

Os testes com o resultados da otimização foram planejados e realizados em 20 corridas, tanto para cargas sem gusa líquida quanto para corridas com gusa líquida. As Figuras 16 e 17 mostram a comparação entre os resultados históricos e os resultados obtidos com os testes para o consumo total de oxigênio, power on, FeO da escória e consumo de energia elétrica.

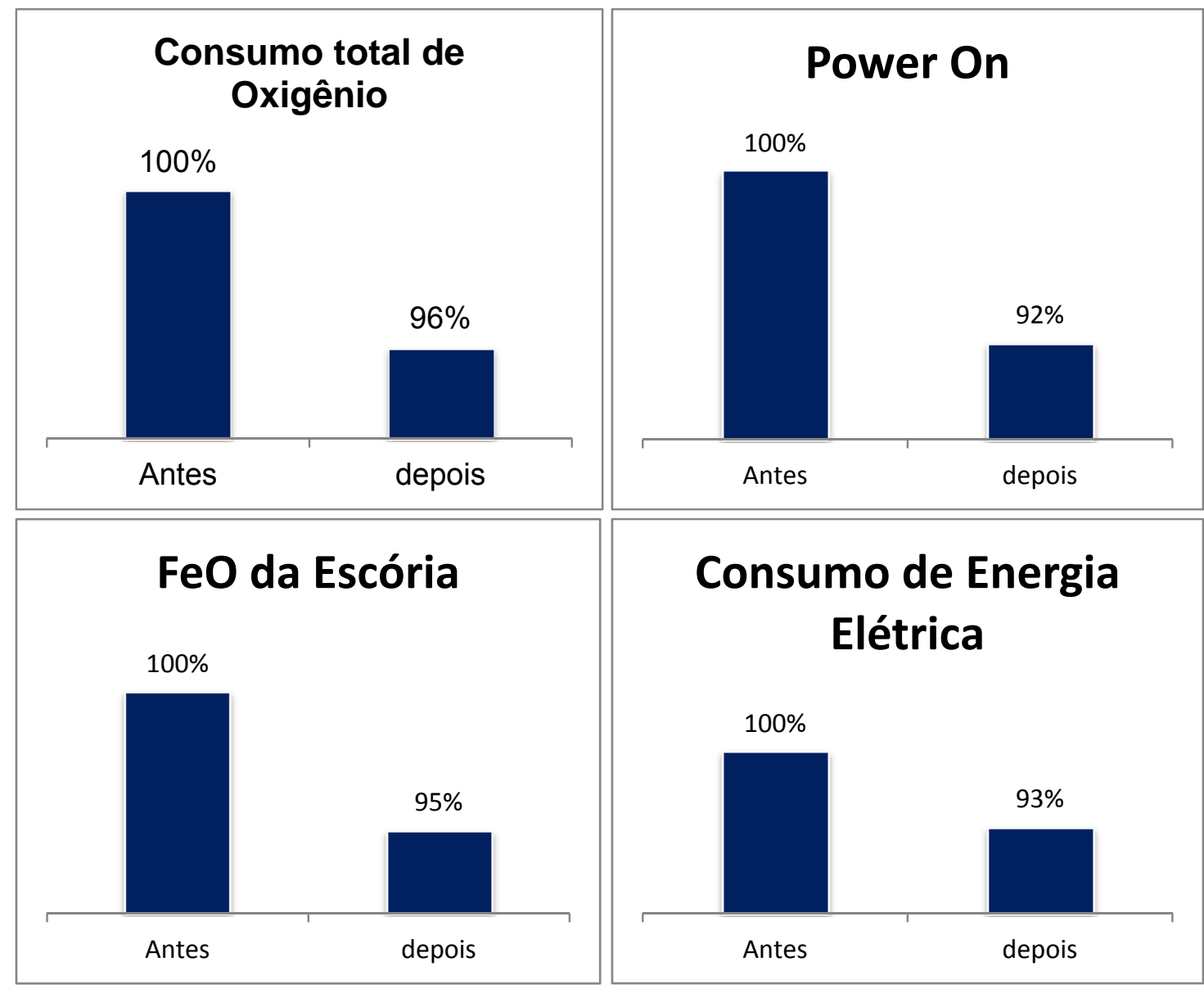

Figura 16. Comparação entre os dados históricos e após a implantação da otimização para corridas sem gusa líquida.

Para corridas sem gusa líquida tivemos redução de 4\% no consumo de oxigênio, $8 \%$ no power on, $5 \%$ no $\mathrm{FeO}$ da escória e $7 \%$ no consumo de energia elétrica.

* Contribuição técnica ao 450 Seminário de Aciaria - Internacional, 25 a 28 de maio de 2014, Porto Alegre, RS, Brasil. 


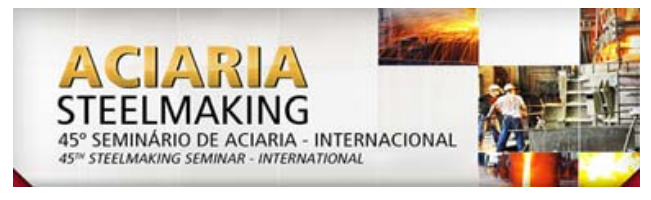

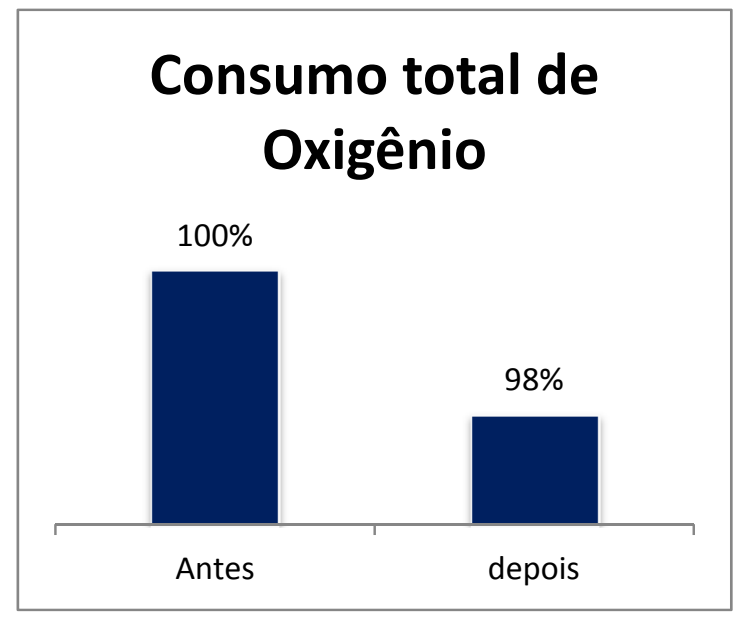

\section{FeO da Escória}

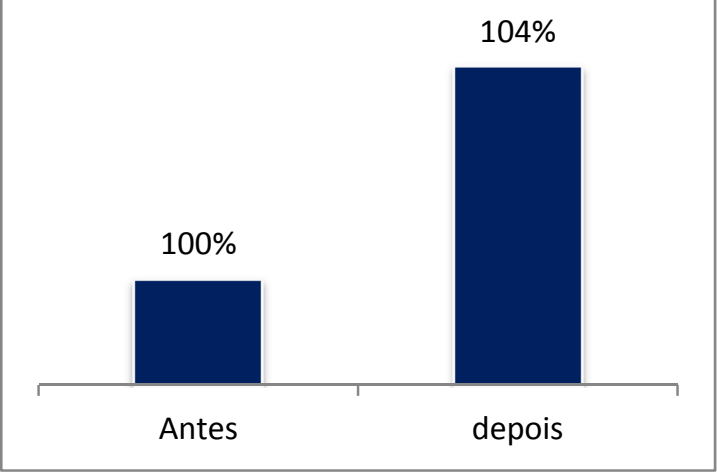

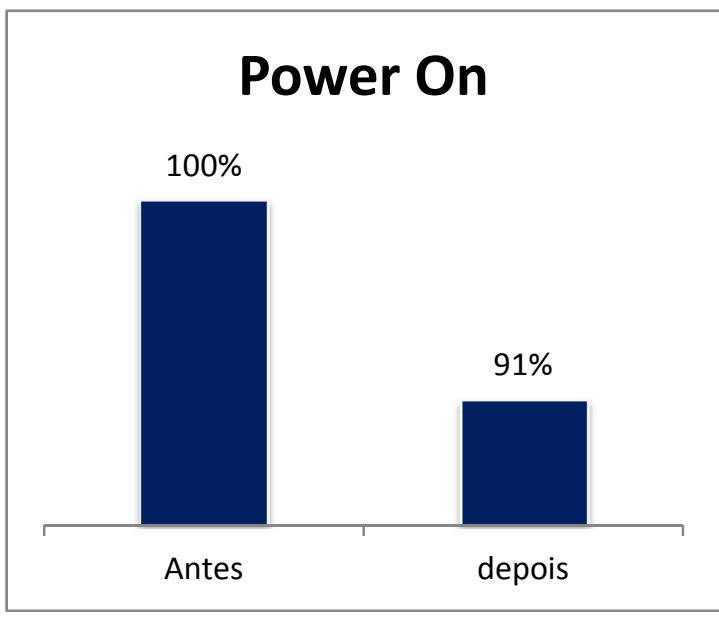

Consumo de Energia Elétrica

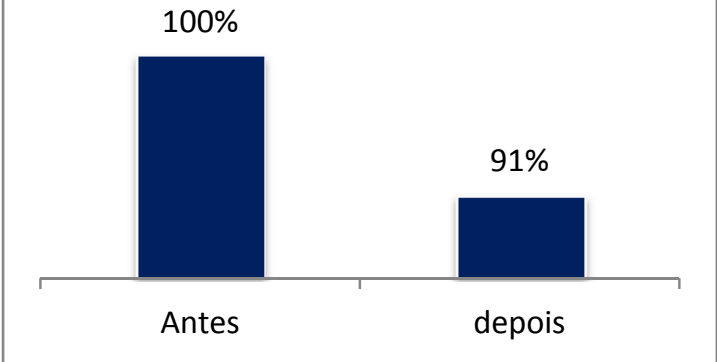

Figura 17. Comparação entre os dados históricos e após a implantação da otimização para corridas com gusa líquida.

Para corridas com gusa líquida tivemos redução de $2 \%$ no consumo de oxigênio, $9 \%$ no Power On, 9\% consumo de energia elétrica e aumento de 4\% no FeO da escória.

\section{CONCLUSÃO}

Com os resultados obtidos na aplicação da ferramenta DOE na fabricação de aço na Sinobras ficou evidente o sucesso do projeto com as seguintes conclusões:

- Tanto para corridas com gusa líquida quantos para corridas sem gusa líquida os resultados foram semelhantes com exceção o teor de FeO para corridas com gusa líquida aumentou $4 \%$ mas não afetou de forma significativa o rendimento metálico e a espumação da escória;

- O modelo proporcionou um menor tempo de processamento tanto para corridas com gusa líquida quanto para corridas sem gusa líquida aumentando assim a produtividade;

- Houve redução no consumo de energia elétrica em $7 \%$ para corridas sem gusa líquida e $9 \%$ para corridas com gusa líquida tendo impacto positivo na sustentabilidade e nos custos da usina.

- Os novos programas de injeção não provocaram, de forma significativa, nenhum efeito indesejado como desgaste de refratário ou diminuição do rendimento metálico.

\footnotetext{
* Contribuição técnica ao 450 Seminário de Aciaria - Internacional, 25 a 28 de maio de 2014, Porto Alegre, RS, Brasil.
} 


\section{REFERÊNCIAS}

1 Adams W, Alameddine S, Bowman B, Nicolas L, Paege S, Stafford P. Factors influencing the total energy consumption in arc furnaces. In: $59^{\text {th }}$ Electric Furnace Conference and $19^{\text {th }}$ Process Technology Conference Update; nov. 2001; Arizona, EUA. 2001. Iron and Steel Society; 2001.

2 Pretorius EB. Fundamentals of the EAF Process. Nova Orleans; 1998.

3 Bihel J. The JetBox burner/injector system for EAF steelmaking. Londres: Millenium Steel; 2006.

4 Chevrand LJS. Aciaria Elétrica. São Paulo: ABM; 2007.

5 Seminário de Aciaria Elétrica UCAR. Rio de Janeiro: Cosigua; 2008.

6 Hopperdiezel P. Curso de Aciaria Elétrica, Módulo Injeção de Gases. São Paulo: ABM; 2007.

7 UCAR. Electric ArcFurnaceDigest. Estados Unidos: UCAR; 2001.

8 Gaskell DR. Introduction to metallurgical thermodynamics. Washington: McGrawhillKogakusha LTD; 1973.

* Contribuição técnica ao 450 Seminário de Aciaria - Internacional, 25 a 28 de maio de 2014, Porto Alegre, RS, Brasil. 